
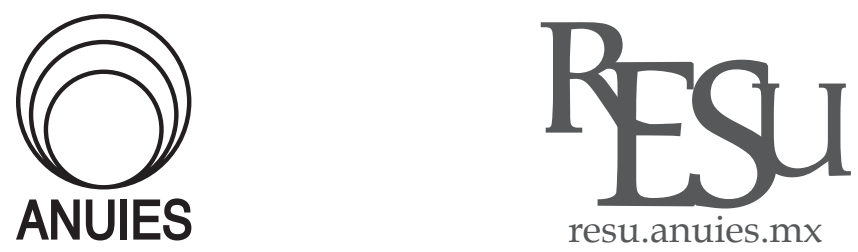

REVITA DEU
EDICACIÓN
SUPERIOR

ARTíCULO

\title{
La educación superior indígena e intercultural en México en 2018: incógnitas, interrogantes y resultados*
}

Intercultural higher education and indigenous in Mexico, 2018:

unknowns, questions and answers

\section{Sylvie Andrée Didou Aupetit**}

* EstuEstudio apoyado por la Secretaria de Educación Pública-Subsecretaria de Educación Superior-Dirección General de Educación Superior Universitaria, convenio pades n. 2017-09-0006-09.

* Correo electrónico: didou@cinvestav.mx

Recibido el 27 de febrero de 2018; aceptado el 10 de septiembre de 2018.

\section{Resumen}

Examinaremos los objetivos de la Educación Superior Intercultural e Indígena (ESII), sus perfiles y modalidades de funcionamiento. Partiremos de la hipótesis que, casi veinte años después de su surgimiento, la ESII incrementó sus grados de institucionalización, en cuanto a regulaciones, gobernanza y recursos. Sin embargo, conserva una condición de sub-campo emergente, caracterizado por tensiones internas y externas y atiende un grupo estudiantil pionero. Pese al interés social y político despertados por la ESII a principios del siglo XXI y la productividad académica de los investigadores que la analizan, las carencias de información sistematizada sobre sus resultados y sus alcances dificultan hacer un balance confiable de su situación.

Palabras clave: Educación superior; interculturalidad; liderazgo indígena; México. 


\begin{abstract}
We will examine the objectives, profiles and governance of Intercultural and Indigenous Higher Education System. Our hypothesis is that, twenty years after its creation, IIHE has improved its institutionalization degree, in terms of regulation, administration and resources. But it is still an emergent field, characterized by internal and external conflicts and it received "pioneer's" students. Albeit the social and political interest for IIHE in the XXI century and the academic productivity of specialized researchers on diverse matters, it is hard to evaluate its results and effects because of the lack of systematized data.
\end{abstract}

Keywords: Higher education; interculturality; indigenous leadership; Mexico

\title{
Introducción
}

$\mathrm{E}$ n 2018, en México, los dispositivos de atención al grupo indígena en educación superior están estructurados en instituciones de base étnica y en programas de educación superior con visos afirmativos en instituciones convencionales. El gobierno federal y los gobiernos estatales financian becas para los estudiantes indígenas y canalizan recursos extraordinarios a las instituciones receptoras, sean ésas convencionales o de base étnica. En paralelo a la expansión de la Educación Superior Intercultural o Indígena (ESII), antropólogos, pedagogos, sociólogos y psicólogos se interesaron en su funcionamiento, por convicción militante o por expertise. Contaron con recursos suministrados por el Consejo Nacional de Ciencia y Tecnología (Conacyt), la Coordinación General de Educación Cultural Bilingüe $(\text { CGEIB })^{1}$ y organismos internacionales. Su productividad (Mateos \& Dietz, 2013) versó esencialmente sobre los proyectos pedagógicos, las condiciones de gestión institucional y los actores principalmente estudiantiles.

En ese artículo nos dedicaremos a analizar la EsI como un sub-campo emergente, y por ende, conflictual: al formar elites pioneras (Bourdieu y Passeron, 1964) y al impulsar la valoración de capitales de autoctonía en la arena educativa nacional, en tanto recursos propios de un grupo (popular o vulnerable) pertene-

\footnotetext{
${ }^{1}$ http:/ / eib.sep.gob.mx/wp-content/uploads/2017/03/INFORMES-DEINVESTIGACI\%C3\%93N.pdf
} 
ciente a un espacio localizado (Coavoux, 2010), consideraremos que promueve proyectos educativos alternos a los dominantes.

Sin embargo, después de una década durante la que la ESI fue una prioridad, está actualmente perdiendo visibilidad. Para demostrarlo, expondremos los ajustes en las políticas públicas de ESII. Nos interesaremos en su estructura, para cumplir con objetivos de inclusión y equidad. Tomando en cuenta que contribuyó a consolidar un colectivo de profesionistas indígenas, concluiremos analizando el estado de los saberes sobre los egresados.

\section{Políticas públicas para la consolidación de un sub-campo educativo}

El surgimiento de la EsII en México es indisociable de la asociatividad de los profesionistas indígenas desde finales de los cuarenta del siglo xx (Maldonado, 2004), y a partir de los 50, de la presencia ingente de los indígenas en el magisterio nacional como promotores educativos y maestros (Martínez, 2011). A partir de los ochenta, las organizaciones indígenas crecieron en número e intensificaron sus movilizaciones. En el campo educativo, varias IES diseñaron programas destinados mayoritariamente a los indígenas: en 1982, en agronomía, el Centro de Estudios para el Desarrollo Rural (CESDER) abrió en Puebla y la Universidad Pedagógica Nacional (UPN) inauguró una licenciatura en educación indígena. En 1990, el Centro de Investigaciones y Estudios Superiores en Antropología Social (CIESAS), el Instituto Nacional Indigenista (INI) y la Dirección General de Educación Indígena (DGEI) de la Secretaria de Educación Pública (SEP) iniciaron una Maestría en lingüística indoamericana. La UPN propuso las licenciaturas en Educación Preescolar y Primaria para el Medio Indígena (LEPEPMI).

En esa misma década, la irrupción en el escenario político nacional del Ejercito Zapatista de Liberación Nacional (EZLN) y la posterior promulgación de cambios constitucionales y leyes sobre derechos indígenas auspiciaron, debido a cuatro filtros, iniciativas dedicadas a elevar la reducida participación de estudiantes indígenas en la educación superior. El primero era socio económico (bajo índice de desarrollo humano del grupo de origen), otro cultural (ausencia de connivencias entre el capital académico y el capital autóctono del grupo indígena), el tercero educativo (deserción temprana de los alumnos indígenas). ${ }^{2} \mathrm{El}$ cuarto era residencial: los indígenas, habitantes de pequeñas localidades rurales,

2 http:/ / www.cdi.gob.mx/programas/2014/ programa-especial-de-los-pueblosindigenas-2014-2018.pdf 
migraron por estudios, durante más tiempo que el resto de la población. Y padecieron los costos financieros y emocionales de ser "foráneos" (Guzmán, 2017).

En la primera década del siglo xxI, Vicente Fox, como el primer presidente que no pertenecía al Partido Revolucionario Institucional (PRI) después de siete décadas de control político ejercido por ese partido, prometió en campaña promover el acceso de la población indígena a la educación superior, y así la canalización de recursos financieros internacionales (créditos, donaciones y cofinanciamientos) se tradujo en un florecimiento de iniciativas gubernamentales y civiles.

El gobierno federal fue el mayor impulsor de la EsI: en distintas modalidades de asociación con las autoridades estatales, creó el subsistema de Universidades Interculturales (las 11 UI), cuya conducción delegó a la Coordinación General de Educación Intercultural y Bilingüe (CGEIB). Agrupaciones indígenas abrieron instituciones orientadas al rescate de la diversidad cultural de los grupos indígenas, al desarrollo de las comunidades y a la formación de líderes (Universidad de la Tierra, en Oaxaca). Otros fueron respaldados por asociaciones étnicas o religiosas como (ISIA) y la Asociación de Universidades Confiadas a la Compañía de Jesús en América Latina (AUSJAL). Las instituciones convencionales de educación superior operaron programas de atención destinados a retener sus estudiantes indígenas. Sectorialmente, las universidades politécnicas incluyeron en sus indicadores de desempeño el incremento del grupo en la matrícula y el suministro de becas a mujeres indígenas (CGUyT, 2015: 36-37 y 70). Los organismos de cooperación internacional financiaron becas, programas de atención a los estudiantes indígenas y de fomento a redes y centros de investigación especializados (Didou, 2013). Objetivos compartidos, aunque a veces discordantes, fueron la inclusión de una población vulnerable, en situación de alteridad y, por ende, el reconocimiento a la diversidad cultural y la interculturalidad, inspirados en prácticas de acción positiva.

\section{Un sub-campo institucionalizado pero con visibilidad menguante}

La apertura de universidades interculturales o con otro perfil fue continua entre 2000 y 2012. Disminuyó después, aunque debido a la mala calidad de la información no podemos afirmar con seguridad que se suspendió del todo en el pasado quinquenio. 
Cuadro 1

Instituciones de educación superior interculturales o indígenas (IESII) México, 2000-2017

2000. Instituto Tecnológico Superior Purépecha, Cherán, Michoacán + Instituto Tecnológico Regional Mixe (a partir de 2014, llamado Instituto Tecnológico del Valle de Etla) + 3 normales superiores indígenas, en Cherán, Michoacán, Zinacantán, Chiapas y Tlacolula, Oaxaca

\begin{tabular}{|c|}
\hline 2002: Colegio Superior para la Educación Integral Intercultural de Oaxaca \\
\hline $\begin{array}{l}\text { 2004: Universidad Intercultural en el Estado de México +Universidad Intercultural de los Pueblos del Sur en } \\
\text { Guerrero + Universidad latinoamericana indígena en Tabasco }\end{array}$ \\
\hline $\begin{array}{l}\text { 2005: Universidad Intercultural de Chiapas+ Universidad Intercultural del Estado de Tabasco +Universidad } \\
\text { Veracruzana Intercultural }\end{array}$ \\
\hline $\begin{array}{l}\text { 2006: Instituto Superior Intercultural Ayuuk, Oaxaca (ISIA) +Universidad Intercultural del Estado de Puebla } \\
\text { + Universidad Intercultural de Guerrero + Universidad Intercultural Indígena de Michoacán + Universidad } \\
\text { Intercultural Maya de Quintana Roo }\end{array}$ \\
\hline 2007: Universidad comunitaria de San Luis Potosí \\
\hline 2011: Universidad Intercultural de San Luis Potosí \\
\hline 2012: Universidad Intercultural del Estado de Hidalgo \\
\hline $\begin{array}{l}\text { Fuente: UI- http://eib.sep.gob.mx/wp-content/uploads/2016/06/Matricula-total-15-16-Octubre-2015.pdf; ISIA- http:// } \\
\text { noticias.universia.net.mx/vida-universitaria/noticia/2014/06/06/1098394/menos-2-poblacion-indigena-ingresa- } \\
\text { educacion-superior.html; UNISUR- http://bibliotecavirtual.clacso.org.ar/ar/libros/osal/osal23/19S3FlorMende.pdf; } \\
\text { Universidad de la Tierra- http://nobeldev.cuaed.unam.mx/bdistancia_ecoesad/contenido/numeros/numero7/ } \\
\text { proyecProg_03.html; Universidad comunitaria de San Luis Potosí- http://sgg.slp.gob.mx/periodicocorr.nsf/698db1bf3277 } \\
\text { 2baa062576ac0068e844/336263011efca76a062577c40059a1f8/\$FILE/Creacion\%20Universidad\%20Comunitaria\%20(14-JUN- } \\
\text { 07).pdf; cSEIIO- http:// www.cseiio.edu.mx/valores.html }\end{array}$ \\
\hline
\end{tabular}

Los propósitos de la ESII (interculturalidad/educación indígena), sus funciones (formación superior para los maestros indígenas/formación de capacidades y conocimientos con propósitos de equidad/reconocimiento a la diversidad) y su estatuto en el sistema nacional de educación superior son heterogéneos. Varían sus recursos y sus orientaciones conforme con sus patrocinadores, aunque todos apoyen mecanismos de empoderamiento de los destinatarios. Funcionan mediante pactos laxamente articulados que, por la diversidad de intereses y posiciones, no sustentan circuitos solidos de apoyo a las instituciones y programas en el sub-campo.

En paralelo, las universidades convencionales abrieron programas de cobertura categorial, enfocados esencialmente a favorecer la retención y el egreso de los estudiantes indígenas. Ésos están todavía en una fase de expansión: la Universidad Autónoma de Guerrero inició en 2013 un programa para indígenas, afrodescendientes, personas con vulnerabilidad e hijos de migrantes: en 2017, registraba a 1507 estudiantes en las áreas de salud (677 en medicina y enfermería), derecho (460), ciencias químicas (250) e ingeniera (120). ${ }^{3}$ La ESII focalizó sus iniciativas sobre la nivelación de los conocimientos académicos de los alumnos indígenas, la generación de conocimientos

${ }^{3}$ https:/ / bajopalabra.com.mx/aumenta-numero-de-estudiantes-indigenas-en-launiversidad-de-guerrero 
científicos y la apertura de carreras vinculadas con la dimensión étnica (licenciatura en Estudios Indígenas entre la UNACH y el Colegio Indio Federado de Saskatchewan (SIFC), Canadá- Ortelli y Sartorello, 2011). En forma minoritaria, algunos establecimientos ofrecieron cupos de ingreso (Universidad Autónoma de Nayarit) o firmaron convenios para la movilidad internacional y estancias de capacitación en asuntos del interés de los indígenas.

\section{Cuadro 2}

Ejemplos de programas de atención a estudiantes indígenas de educación superior, según objetivo, México, 2000-2017

\begin{tabular}{|c|c|}
\hline Denominación del programa & Organismo patrocinador \\
\hline \multicolumn{2}{|l|}{ a. Programas de becas para estudios completos } \\
\hline Fortalecimiento académico para indígenas & Conacyt (cobertura nacional) \\
\hline $\begin{array}{l}\text { Apoyos complementarios para mujeres indígenas } \\
\text { becarias del Conacyt }\end{array}$ & Conacyt (cobertura nacional por género) \\
\hline $\begin{array}{l}\text { Incorporación al posgrado de mujeres indígenas para } \\
\text { el fortalecimiento regional }\end{array}$ & Conacyt (cobertura nacional por género) \\
\hline $\begin{array}{l}\text { Educación superior y apoyo a titulación para } \\
\text { estudiantes indígenas que cursen sus estudios de nivel } \\
\text { superior }\end{array}$ & $\begin{array}{l}\text { Comisión Nacional para el Desarrollo de los } \\
\text { Pueblos Indígenas -CDI (cobertura nacional en } \\
\text { educación superior) }\end{array}$ \\
\hline Becas para estudiantes indígenas & $\begin{array}{l}\text { Gobiernos estatales Estados de Chihuahua, } \\
\text { México, Guerrero, Jalisco, Oaxaca o Sonora } \\
\text { (cobertura por entidad federativa en educación } \\
\text { superior y medio superior) }\end{array}$ \\
\hline Sistema de becas para estudiantes indígenas & $\begin{array}{l}\text { UNAM (Cobertura institucional en preparatoria y } \\
\text { educación superior) }\end{array}$ \\
\hline $\begin{array}{l}\text { Programa internacional de becas de posgrado para } \\
\text { indígenas }\end{array}$ & $\begin{array}{l}\text { CIESAS-Conacyt (PROBEPI)-(cobertura nacional en } \\
\text { posgrado) }\end{array}$ \\
\hline $\begin{array}{l}\text { Estímulos Económicos para Estudiantes de } \\
\text { Comunidades Indígenas }\end{array}$ & $\begin{array}{l}\text { Universidad de Guadalajara (cobertura } \\
\text { institucional en preparatoria y educación } \\
\text { superior) }\end{array}$ \\
\hline International Fellowship Program (2001-2013) & $\begin{array}{l}\text { CIESAS-Fundación Ford (cobertura nacional en } \\
\text { posgrado }\end{array}$ \\
\hline \multicolumn{2}{|l|}{ b. Acciones de retención } \\
\hline Pathways (2002-2012) & $\begin{array}{l}\text { Fundación Ford/ANUIEs (Cobertura institucional } \\
\text { múltiple en licenciatura) }\end{array}$ \\
\hline $\begin{array}{l}\text { Talleres de preparación para estudiar un posgrado en } \\
\text { Estados Unidos }\end{array}$ & COMEXUs (cobertura nacional en posgrado) \\
\hline Programa de Desarrollo de Comunidades Indígenas & $\begin{array}{l}\text { Instituto Tecnológico de Cajeme (cobertura local/ } \\
\text { estatal) }\end{array}$ \\
\hline \multicolumn{2}{|l|}{ c. Becas para estancias de capacitación } \\
\hline $\begin{array}{l}\text { Título de Experto en "Pueblos Indígenas, derechos } \\
\text { humanos, y cooperación internacional" }\end{array}$ & $\begin{array}{l}\text { Universidad Indígena Intercultural (UII) y } \\
\text { Universidad Carlos III (España) }\end{array}$ \\
\hline $\begin{array}{l}\text { Programa de becas para el liderazgo social en América } \\
\text { Latina y El Caribe }\end{array}$ & Fundación Kellog \\
\hline \multicolumn{2}{|c|}{$\begin{array}{l}\text { Fuente: https://www.conacyt.gob.mx/index.php/becas-y-posgrados/becas-nacionales/programa-de-fortalecimiento- } \\
\text { academico-para-indigenas; + http://www.cdi.gob.mx/focalizada/2016/paei/index.html; +http://seduc.edomex.gob. } \\
\text { mx/becas_iindigenas; http://guerrero.gob.mx/articulos/convocatoria-programa-de-becas-a-estudiantes-indigenas-y- } \\
\text { afromexicanos-2017/; https://info.jalisco.gob.mx/gobierno/programas apoyo/15945; http://cedis.sonora.gob.mx/images/ } \\
\text { contenidos/Program-Becas-Ind2017.pdf; + http://becasindigenas.ciesas.edu.mx/+ http://www.ntrguadalajara.com/post. } \\
\text { php?id_nota=70755; + http://www.itesca.edu.mx/estudiantes/comunidades_indigenas.asp; + http://www.comexus. } \\
\text { org.mx/taller_estudiantes_indigenas.html; + http://www.unich.edu.mx/programa-de-becas-para-el-liderazgo-social-en- } \\
\text { el-caribe-y-latinoamerica-fortaleciendo-a-los-lideres-comunitarios-en-mexico-y-haiti-patrocinado-por-la-fundacion-w-k- } \\
\text { kellogg/8242 }\end{array}$} \\
\hline
\end{tabular}


En su conjunto, esos programas e instituciones acercaron los proveedores del servicio educativo y los usuarios: así, en 2015-2016, las ui tenían campus y unidades en 39 municipios y localidades con alta densidad de población indígena. Sin embargo, pese a ese redespliegue territorial, la atención prestada a la educación superior indígena se debilitó en las escalas gubernamental e institucional. El concepto dominante en las políticas públicas de atención social fue, en el pasado sexenio, el de "población vulnerable", que engloba personas con discapacidad, poblaciones indígenas, poblaciones rurales, migrantes o estudiantes que han abandonado el sistema educativo.

En un contexto en el que los programas de base étnica fueron subsumidos en programas de extenso radio, destinados a grupos heterogéneos que comparten como rasgo común el estar en situación de precariedad, las universidades desvincularon crecientemente el aumento de la equidad de la incorporación preferente del grupo indígena. En 2016, sólo la Universidad Juárez del Estado de Durango, la Universidad de Nayarit, la Universidad Autónoma de Chiapas, la Universidad de Quintana Roo, los institutos tecnológicos de Salinas Cruz y Superior de la Costa Chica y las ur Maya y de Guerrero presentaron propuestas, focalizadas sobre esos grupos, de las 70 aprobadas por el Programa para la Inclusión y Equidad Educativa de la SEP. ${ }^{4}$ Esa situación pone en duda el futuro de las experiencias de rescate de las lenguas indígenas, adaptación de los procesos formativos y didácticos, codificación e incorporación de los conocimientos autóctonos a la vida científico y pedagogías interculturales, indispensables para "mejorar las condiciones sociales de los pueblos indígenas [y] reconocer, re-encontrarse, re-valorar, re-aprender y readaptar procesos cognitivos que conduzcan a la generación de conocimiento, a partir de la interacción de la cultura originaria con el método científico" (Rosado-May, 2017: 221).

\section{La ESII y la cobertura de la población indigena: una expectativa sin sustento estadístico}

Uno de los resultados sobre el que concuerdan los especialistas es que la ESII elevó los porcentajes de acceso y permanencia de la población indígena, aun cuando ésos siguen estando por debajo de las tasas promedio nacionales. Conforme con los datos del Censo del 2010:

${ }^{4}$ http://www.dgesu.ses.sep.gob.mx/documentos/DSA\%20gobmx/pdf/2do.Trim_S244. pdf 
La población indígena ha alcanzado $66 \%$ de los resultados educativos logrados por la población no-indígena. En otras palabras, la brecha en logros educativos que separa a los indígenas de los no-indígenas en México es de $34 \%$ [...] La mayor inequidad y los menores niveles de escolaridad para ambas poblaciones se observan en la educación superior. El valor del indicador de equidad de Escolaridad Superior es de 0.22: los indígenas han alcanzado $22 \%$ de la escolaridad superior lograda por los no-indígenas. Uno de cada cinco no-indígenas mayores a 24 años ha concluido al menos un año de estudios superiores $(19 \%)$, mientras que solamente cuatro de cada cien indígenas lo ha hecho (Fundación Idea, 2013:23-27).

Aunque la disminución relativa de la brecha de escolaridad sea uno de los ejes del discurso sobre la EsII y un indicador de evaluación, llama la atención la dificultad perenne para acceder a cifras fidedignas sobre los estudiantes indígenas. Los expertos suelan señalar un mejoramiento porcentual de sus tasas de acceso (de $1 \%$ a entre 2 y $4 \%$ ). Pero no precisan si lo refieren al grupo indígena por auto-adscripción, residencia, exo-identificación, dominio o comprensión de una lengua indígena, aun cuando el indicador seleccionado incide en el cálculo de su tamaño, al igual que para la población indígena en general . Por ende, las necesidades de información sobre la matricula indígena en la educación superior son todavía apremiantes.

Para apreciar cuantos estudiantes indígenas están matriculados en la educación superior, disponemos de cifras sueltas cuya confiabilidad no siempre es argumentada, principalmente en prensa o en rankings. Por ejemplo, en 2015-16, el ranking America Economy acreditó 50 instituciones que registraron 9709 estudiantes indígenas. ${ }^{5}$ Sin embargo, esas cifras no corresponden con las reportadas por fuentes oficiales, probablemente porque fueron construidas con base en la definición más favorable para una buena valoración de cumplimiento del indicador. Aplicar un criterio de auto-adscripción en lugar de uno del dominio (pasivo/activo) de la lengua incide en el volumen de estudiantes indígenas, conforme con dinámicas de reetnificación y de instrumentalización de la condición étnica, en respuesta a medidas compensatorias.

Por sector, según las autoridades, en 2014-15, las universidades tecnológicas y politécnicas agrupaban a 6309 estudiantes indígenas (Arreola, 2015). En 2015-2016, las interculturales reunían a 14007 alumnos (CGEIB, 2015), las normales a 8841 y la UPN a 8413 en las licenciaturas para el medio indígena

\footnotetext{
${ }^{5}$ En 2015, la población total indígena reunía 12025947 individuos, la hablante de lengua indígena 7382725, la monolingüe 909356 y la auto-adscrita 25694928 (Rojas et al., op. cit.)
} 
(Rojas et al., 2017). En 2016-2017, según el formato 911 de la SEP, $^{6}$ hablaban una lengua indígena 5071 alumnos en las interculturales, 45830 alumnos de licenciatura y TSU, inscritos esencialmente en el sector público y en la modalidad presencial. Su número había aumentado en un $21.6 \%$ en comparación con 2012-2013,7 aunque seguían representando en 2016-2017 un porcentaje bajo de la matrícula total $(1.12 \%)$. Por institución, se concentraban en establecimientos con vocación agraria (Universidad Autónoma de Chapingo y Antonio Narro), de formación de docentes (UPN) y en algunos institutos tecnológicos: representaban entre un tercio y casi dos tercios de la matricula total en los de Calkini y Holpechen (Campeche), de la Montaña (Guerrero), de San Miguel El Grande (Oaxaca), de Tecomatlan (Puebla), de Felipe Carrillo Puerto (Quintana Roo) o de Valladolid y del Sur del Estado de Yucatán (Yucatán). Esa distribución territorial debería llevar a definir si apoyar mejores prácticas de atención a las matriculas indígenas en las instituciones con experiencia acumulada para elevar los promedios de titulación o bien incorporar a más establecimientos para incrementar la tasa de acceso.

Ignoramos, en cambio, cuántos estudiantes indígenas reciben becas. Pocos programas reportan cifras. Los estados de Jalisco y Sonora registran 861 y 501 becarios indígenas, la UDG 738, la UNAM 850, el Conacyt 468 en posgrado, el Probepi-CIESAS 39 (2016-17), la SEP $1725^{8}$ y la CDI 2580 (2015-16), ${ }^{9}$ pero no desglosan a los beneficiarios por nivel ni separan los indígenas de los afrodescendientes. La escasa información sobre los alcances y resultados de los programas de becas impide medir sus contribuciones al mejoramiento en la igualdad de participación y a la eficiencia en la redistribución de oportunidades.

\footnotetext{
${ }^{6}$ Datos proporcionados a 16 de febrero 2017 por el Mtro. Guillermo Antonio Reyna Figueroa, Subdirector de Sistematización y análisis de indicadores, SEP_DGESU.

${ }^{7}$ La matrícula indígena parece haber crecido más en las universidades convencionales que en las interculturales. Esa apreciación deriva quizás del hecho de que los registra una mayor proporción de las convencionales que anteriormente.

${ }^{8}$ https:/ / www.coneval.org.mx/Evaluacion/Documents/EVALUACIONES/ EED_2014_2015/CDI/S178_PAEI/S178_PAEI_IE.pdf

${ }^{9}$ Es probable que parte de esos alumnos sean reportados entre los beneficiarios de los programas de becas indígenas [http:/ / www.cdi.gob.mx/focalizada/2016/paei/index. html].
} 


\section{¿Reconfigurar las elites y/o redistribuir oportunidades a un grupo específico de población?: un tema en discusión}

En 2015, los porcentajes de población entre 25 y 64 años de edad con educación superior completa eran de $18.7 \%$ en el país, pero bajaban a $6.6 \%, 4.7 \%$ y $9.7 \%$ en la población indígena total, en la HLI y en la auto-adscrita, respectivamente (Rojas, op. cit., tabla 3: 18). Los que tenían posgrado eran todavía menos: "Sólo 11 mil 711 hablantes de lengua indígena contaban con estudios de maestría para el año 2010, y dos mil 538 contaban con estudios de doctorado, lo cual representa 1.8 por ciento de todas las personas con doctorado en el país" (Nava, 2016). Si bien los profesionistas indígenas constituían una fracción mínima de su grupo de origen, inferior al promedio nacional, se considera que la ESI contribuyó a expandir el grupo, en términos cuantitativos y, sobre todo, cualitativos, al modificar los perfiles de formación de sus integrantes. De allí, el interés reciente de los especialistas por realizar estudios de seguimiento de egresados y analizar una "neo-intelectualidad" indígena, integradas por técnicos, expertos, profesionales, intelectuales y políticos.

Todos ellos ocupan posiciones de elite en ciernes, con distintos rangos de visibilidad. Sus ventajas son superiores a las de los demás integrantes de su grupo de procedencia, pero su estatuto es marginal en comparación con el de las elites nacionales tradicionales, conforme con el fenómeno general de caleidoscopización de las elites (Estrada-Puello, 2005). Sociólogos y antropólogos (Gutiérrez, 2012; López, 2016) plantearon como rasgos de identidad de sus integrantes haber concluido estudios superiores, dominar conocimientos occidentales y nativos y producir aportes, socialmente validados, sobre lo indígena:

En ALC, la posibilidad de acceder a la Es produce una "capa creciente de intelectuales indígenas quienes, en busca de nuevos modelos para la sociedad civil, experimentan con modos nativos y occidentales de conocimiento con el fin de fortalecer las reivindicaciones étnicas a los niveles regional y nacional. Además de su contribución como activistas, este nuevo sector dentro de la sociedad indígena está tomando parte en los diálogos étnicos a través de la publicación de sus propias investigaciones sobre aspectos de la sociedad nativa y su relación con la sociedad dominante, frecuentemente dentro de los campos de la antropología y la historia. (Rappaport, 2007: 615). 
Los integrantes de esos colectivos, principalmente del intelectual, por sus experiencias escolares y sus posiciones respecto de la cuestión indígena, tuvieron fricciones con líderes arraigados en sus comunidades y con intelectuales convencionales, ubicados en esquemas profesionales de diálogo y negociación con las instancias gubernamentales y los organismos a cargo de las políticas indigenistas (Baronnet, 2017). Abogan en efecto por dejar atrás los esquemas integracionistas y de asimilación clientelista históricamente predominantes. Pretenden socializar y difundir perspectivas críticas acerca de las necesidades de las poblaciones de las que se asumen como voceros. Convocan a desarrollar un pensamiento emancipatorio, a luchar por la defensa de sus derechos. Encarnan figuras radicales y de disidencia cuya legitimidad de palabra depende de su condición étnica. Junto con comunidades epistémicas (antropólogos, psicólogos y pedagogos), expertos y autoridades sectoriales, forman opiniones acerca de las misiones, estructuras y alcances de la ESII, reflexionando sobre:

a) Las funciones de la ESII en tanto promotora de la equidad, de la inclusión o de la generación de capacidades autónomas, en un espectro valoral que abarca de la solidaridad social al pago de una deuda histórica;

b) sus orientaciones y sus adscripciones en el campo de la educación superior según parámetros propios o generales de funcionamiento;

c) sus propósitos (acción afirmativa, meritocracia auxiliada o autoafirmación);

d) sus objetivos: ampliación de las elites sociales procedentes de grupos sociales vulnerables versus rescate de la dignidad indígena;

e) su potencial transformativo en las comunidades, en las trayectorias familiares, en los sujetos individuales;

f) la generación de redes estratégicas con fines de vinculación y de empoderamiento.

Abonan así al debate abierto sobre los fines de la ESII en función de si se orienta mejorar la equidad de acceso y egreso de los individuos a la educación superior o bien a cuestionar "el gran proyecto de la modernidad desde perspectivas epistemológicas no occidentales" (Bastian, 2015), mediante labores de concientización y de movilización política.

$\mathrm{Su}$ activismo expresa un proceso en curso para fabricarse una identidad social: para ello, emiten discursos sobre lo indígena, legitimados profesionalmente por la ocupación de puestos académicos, en instituciones universitarias. La elaboración de enfoques tales como la etnogénesis, la comunalidad y la descolonización (Canales, 2014), el asumir responsabilidades críticas y movilizadoras, son parte de una dinámica de "autodefinición" y de estrategias de posicionamiento de un colectivo frágil, por su déficit de reconoci- 
miento social fuera de ciertos espacios. Revela una pugna por mantener en la agenda nacional la cuestión indígena, aun a costa de posiciones que suscitan reparos, como la de contar con una expertise por el hecho de pertenecer (Puello-Socarras, 2009).

En esa perspectiva, seguir documentando las trayectorias de profesionalización de los egresados indígenas, sus interacciones con sus grupos de origen y con la sociedad nacional, es crucial. ¿Constituyen ellos un grupo de poder, en el cruce de las dimensiones políticas, culturales y académicas, o manifiestan una coalición discursiva encaminada a empujar el reconocimiento de una identidad étnica políticamente redituable? ¿Encarnan un imaginario identitario, fundamentado en la elaboración de ciudadanías resilientes y resistentes? Por ahora, los pocos estudios realizados evidencian una diferenciación de sus nichos de empleo, en donde capitalizan o no su pertenencia indígena, pero no arrojan luz sobre sus roles, en tanto agentes en disputa. No permiten establecer, de manera concreta, cómo transforman el legado de sus antecesores (Llanes, 2008) o reelaboran su herencia. No esclarecen si la ESII forma a recursos humanos altamente calificados (es decir a profesionistas eficientes) o si logra su cometido de preparar a líderes que defiendan los derechos indígenas, desde la comunidad o desde afuera, robusteciendo capacidades colectivas y personales de intervención social o política.

Los estudios de seguimiento de egresados (Martel, 2017; Mateos, 2016) o las ego-historias ${ }^{10}$ confirmaron todos que la noción de liderazgo requiere ser problematizada. Plantean en efecto interrogantes sobre la libertad de elección del sujeto versus los compromisos comunitarios que se le asigna. En efecto, ¿el requerimiento del liderazgo participa de una voluntad compartida de codesarrollo de grupos vulnerables o es una manifestación más de un proceso de esencialización de lo indígena versus identidades líquidas o alternas? ¿Es la preconización de solidaridad étnica un imperativo moral o una prescripción política? ¿Es la manifestación de una visión monolítica y aprioristica de un grupo social o de una utopía conforme a la que se pretende auspiciar la responsabilidad de los más educados hacia los que lo son menos?

Por lo pronto, pese una discriminación laboral ampliamente denunciada (Unich, 2010), esos estudios de seguimiento revelan que, según los nichos de profesionalización y las carreras estudiadas, las habilidades asociadas con la condición indígena o bien son altamente valoradas (radio indígena/traductores jurídicos) o bien se disuelven en beneficio de la posesión de habilidades técnicas. Faltaría sin embargo que integren en su universo de estudio dimen-

\footnotetext{
${ }^{10}$ Aquí estamos, n. 15, Julio 2011, https://ciesas.wordpress.com/2012/03/25/revistaaqui-estamos-del-programa-de-becas-para-estudiantes-indigenas/
} 
siones menos clásicas o técnicas, tales la formación de nuevos intelectuales y la autodefinición de sus roles y de sus responsabilidades, no sólo en términos culturales sino políticos. Un estudio reciente sobre los mapuches, en Chile, corrobora en efecto la relevancia de esas dimensiones:

Otras diferencias tienen relación con un hilado conceptual más fino, en que aparecen nociones abiertas e inclusivas respecto de lo que sería la intelectualidad indígena, que refiere a posiciones de liderazgo y capacidad de elaborar miradas y discursos críticos no restringidos a la escritura, aunque se distingue esta práctica al interior de este segmento de intelectuales, mientras que otras enfatizan o se refieren casi exclusivamente a esta última modalidad, llegando a establecer una dicotomía entre profesionales e intelectuales (Zapata \& Rojas, 2017: 331).

En consecuencia, habría que fortalecer, en los estudios sobre la ESII, líneas de investigación sobre profesionistas indígenas, sus auto-percepciones, sus compromisos y sus mecanismos representativos (red, asociación, ong/sociedad, círculos, grupos de reflexión o de intervención) para producir conocimientos especializados, resolver problemas, ganar superficie social y acceder a recursos y a posiciones.

\section{Conclusiones}

\section{La ESII: Una institucionalización en tensión}

Al iniciar la ESII, los especialistas discutieron la durabilidad y la portabilidad de sus carreras, y la participación, autónoma o supeditada, de los actores indígenas en los asuntos pedagógicos y en la toma de decisiones. En la fase presente de estabilización, critican más bien sus condiciones normativas y financieras de implementación, fracturadas entre un modelo institucional innovador y una estructura administrativa rutinaria (González(a), 2017:48-49).

Varios rectores fundadores de las UI argumentaron que sus dificultades para crecer proceden de presupuestos insuficientes, de exigencias regulatorias inadecuadas por parte de la Secretaria de Educación Pública (SEP) y de la intromisión de los gobiernos estatales en la vida académica (Fábregas, 2017; Cabrera, 2017; González(b), 2017; Salmerón, 2017). Los obstáculos para acreditar los procesos de enseñanza e investigación y reconocer los títulos expedidos por la ESII erosionan la legitimidad de los establecimientos. Las cargas de enseñanza dificultan la realización de tareas como la investigación. 
El perfil de la carrera docente incide negativamente en la fidelización de las plantillas. Originalmente, la pregunta “¿Quiénes enseñan?” aludió a los criterios étnicos, lingüísticos o académicos para la contratación, y a la asignación de cargas de enseñanza e investigación. Hoy remite a la necesidad de insertar a los profesores de la ESII en programas de sobresueldos y de legitimación profesional como el Sistema Nacional de Investigación (SNI) y el Programa para el Desarrollo Profesional Docente (Prodep), ${ }^{11}$ en un contexto de presiones fuertes para estandarizar los comportamientos y modos de funcionamiento conforme con un mandato homogeneizador vehiculado por las políticas públicas.

En un contexto de financiamiento extra-presupuestal amarrado a sus preconizaciones por parte del gobierno que induce todas las instituciones de educación superior a adaptarse a los mismos estándares de desempeño, son restringidos los espacios para la diferencia y la autonomía. Una temática a indagar concierne la profundidad y la extensión de los cambios (irreversibles o superficiales) que produjo la consolidación de la EsII, más allá de que la obtención de un grado universitario mejore a corto o mediano plazo las oportunidades de los individuos procedentes de medios sociales desfavorecidos. Al respecto y en el caso del IFP: “[...] pese a la labor de seguimiento de exbecarios realizada desde 2005 por la Coordinación del IFP México en el CIESAS, quedaron abiertas preguntas centrales sobre el impacto a largo plazo que el programa tendría en la vida personal de sus becarios y la proyección social más amplia de sus acciones"(Navarrete, 2017).

Otra temática a estudiar es la intervención de los actores indígenas y no indígenas en la operación de la ESII. A la disparidad entre, en términos genéricos, la escuela soñada por las comunidades indígenas y la reclamada por los padres de familia, se superpone otra, entre la escuela con un vínculo orgánico con las comunidades y la diseñada por expertos con miras a una inclusión eficiente. Quedan todavía sin respuesta, en México, a una disyuntiva formulada por Gros (2012:11) sobre “[...] cómo construir entre universalismo y comunitarismo un espacio de convivencia que permita reconocer los derechos culturales propios de quienes se identifican a sí mismos como indígenas, he-

${ }^{11}$ En 2018, el SNI registraba en su padrón de beneficiarios a 29 docentes-investigadores de las uI entre sus 28633 beneficiarios: 9 en Chiapas y 9 en Puebla, 5 en el Estado de México, 4 en Michoacán y 2 en Quintana Roo. https://www.conacyt.gob.mx/index. $\mathrm{php} /$ el-conacyt/sistema-nacional-de-investigadores. El Prodep, más enfocado a la docencia, reconocía 38 cuerpos académicos, dos consolidados, cinco en consolidación y el resto en formación, con unos 180 participantes. https:// promep.sep.gob.mx/ca1/ firmadopalabraMEJORA.php?RELOAD=1 
rederos de los primeros habitantes, sin encerrar por tanto a los individuos y a los grupos en fortalezas comunitarias".

\section{Referencias}

Arreola, H. (2015). Aportes para fortalecer la innovación en los sectores y las regiones. Recuperado de: http://www.foroconsultivo.org.mx/eventos_realizados/ Aportes_para_fortalecer_la_innovacion/presentaciones/Panel_6/Ing.hector_arreola.pdf

Baronnet, B. (2017). Estrategias alternativas de educación en las luchas de los pueblos originarios en México. Educação \& Sociedade, 140 (38), 689-704. Recuperado de: http://www.scielo.br/pdf/es/v38n140/1678-4626-es-38-140-00689. pdf

Bastian, I. (18 de mayor 2015). Surcos y redes. Conversación sobre (y con) intelectuales indígenas en México. Recuperado de: http://notaalpie.org/2015/05/ surcos-y-redes-conversacion-sobre-y-con-intelectuales-indigenas-en-mexi$\mathrm{co} /$

Bourdieu, P. y J. C. Passeron (1964). Les héritiers. Paris, ed. Minuit.

Cabrera, H. (2017). La Educación Superior Intercultural en Tabasco: entre la Teoría, el Contexto y su Financiamiento. En González, F. Rosado-May, F. \& Dietz, G. (2017). La gestión de la Educación Superior Intercultural en México (pp. 89120). México: Universidad Autónoma de Guerrero A.C.

Canales, P. (2014). Intelectualidad indígena en América Latina: Debates de descolonización, 1980-2010. Universum 29(2), 49-64. Recuperado de: https:/ / scielo. conicyt.cl/scielo.php?script=sci_arttext\&pid=S0718-23762014000200005

Coavoux S. (2011). Mobilité / autochtonie. Sur la dimension spatiale des ressources sociales. Regards sociologiques (40). Recuperado de: http://journals. openedition.org/lectures/1281\#text

CGUT (2015). Programa institucional de Desarrollo, 2013-2018. México, SEP. Recuperado de: http://cgut.sep.gob.mx/PIDUP/Programa\%20Institucional\%20 de\%20Desarrollo\%02013-2018\%20Politecnicas.pdf

Didou, S. (2013). Cooperación internacional y educación superior indígena en América Latina: constitución de un campo de acción. Revista Iberoamericana de Educación Superior, 4 (11). Recuperado de: https://ries.universia.net/article/ view/126/304

Estrada, J. \& Puello-Socarrás, J. (2005). Élites, intelectuales y tecnocracia. Calidoscopio contemporáneo y fenómeno latinoamericano actual. Colombia Internacional (62), 100-119. Recuperado de: http://www.redalyc.org/articulo. oa?id $=81206207$

Fábregas, A. (2017). la Universidad Intercultural de Chiapas: una experiencia interrumpida. En González, F.; Rosado-May, F. \& Dietz, G. (2017). La gestión de la Educación Superior Intercultural en México (pp. 65-88). México : Universidad Autónoma de Guerrero A. C. 
Fundación IDEA (2013). Índice de equidad educativa indígena. México: Naciones Unidas. Recuperado de http://www.fundacionidea.org.mx/educacion/indicede-equidad-educativa-indigena/152

González(a), F. (2017). Procesos Sociales y Educación. La Universidad Intercultural del Estado de México. En González, F.; Rosado-May, F. \& Dietz, G. (2017). La gestión de la Educación Superior Intercultural en México (pp. 33-64) México: Universidad Autónoma de Guerrero A. C.

González(b), F. (2017). La Universidad Intercultural del Estado de Guerrero; o la travesía de un proyecto frustrado. En González, F.; Rosado-May, F. \& Dietz, G. (2017). La gestión de la Educación Superior Intercultural en México (pp. 205-252) México: Universidad Autónoma de Guerrero A.C.

Gros C. (2012). Políticas de la etnicidad; identidad, estado y modernidad. Colombia: Instituto Colombiano de Antropología e Historia. Recuperado de: http:/ / biblioteca.icanh.gov.co/DOCS/MARC/texto/306.08998G877p.pdf

Gutiérrez, N. (2012). Mitos nacionalistas e identidades étnicas: los intelectuales indígenas y el Estado Mexicano. México: UnAM. Recuperado de: http:/ / ru.iis.sociales.unam.mx/jspui/bitstream/IIS/4417/1/Mitos\%20nacionalistas $\% 20$ e $\% 20$ identidades\%20etnicas.pdf

Guzmán, C. (2017). Las nuevas figuras estudiantiles y los múltiples sentidos de los estudios universitarios. Revista de la Educación Superior (182) 71-87 http:/ / publicaciones.anuies.mx/pdfs/revista/Revista182_S1A5ES.pdf

Llanes, G. (2008). Interculturalización fallida. Desarrollismo, neoindigenismo y universidad intercultural en Yucatán. Trace (53) 49-63. Recuperado de: http:/ / www.redalyc.org/pdf/4238/423839509004.pdf

López, F. (2016). El pensamiento indígena contemporáneo emancipatorio. Contralínea. Recuperado de: http://www.contralinea.com.mx/archivo-revista/2016/08/11/el-pensamiento-indigena-contemporaneo-emancipatorio/

Maldonado, E. (2004). Desde las voces cantarinas al testimonio indígena. UAM. Recuperado de: https://www.azc.uam.mx/publicaciones/tye/vocescantarinas. htm

Martel, M. (2017). Social Justice and Sustainable Change: The Impacts of Higher Education. IIE-Fundación Ford. Recuperado de: https:/ / www.acu.ac.uk/about-us/ blog/can-individual-outcomes-lead-communal-impacts-measuring-socialchange-using-longitudinal-studies

Martínez, R. (2011). La formación de los profesionistas bilingües indígenas en el México contemporáneo. Perfiles educativos (33). 250-261. Recuperado de: http:/ / www.scielo.org.mx/scielo.php?script=sci_arttext\&pid=S018526982011000500021\&lng=es\&tlng=es.

Mateos, L. et al. (2016). ¿Saberes-haceres interculturales? Experiencias profesionales y comunitarias de egresados de la educación superior intercultural veracruzana. Revista mexicana de investigación educativa, 21 (70), 809-835. Recuperado de: http://www.scielo.org.mx/scielo.php?script=sci_arttext\&pid $=$ S1405-66662016000300809

Mateos, L. S. \& Dietz, G. (2013). “Universidades Interculturales en México”, en Bertely, M.; Dietz, G. \& Díaz, M. G. (Coords.). Multiculturalismo y educación 2002-2011. México: comie-ANUIEs: 349-381. 
Nava, A. (8 agosto 2016). Participación de los pueblos indígenas en la creación científica. Conacyt Prensa. Recuperado de: http://www.conacytprensa.mx/ index.php/ciencia/humanidades/9510-creacion-cientifica-pueblos-indigenas-dia-internacional

Navarrete, D. (2017). Resultados de una acción afirmativa en un contexto complejo: El caso de becas de posgrado para indígenas. Nexos. Recuperado de: https:/ / educacion.nexos.com.mx/?p=782

Ortelli, P. \& S. Sartorello (2011). Jóvenes universitarios y conflicto intercultural: Estudiantes indígenas y mestizos en San Cristóbal de Las Casas, Chiapas. Perfiles educativos, 33 (spe), 115-128. Recuperado de: http:// www.scielo.org. $\mathrm{mx} /$ scielo.php?script=sci_arttext\&pid=S0185-26982011000500011

Puello-Socarras, J. (2009). Política -qua experticia- Élites intelectuales, tecnocracia, think-tanks, Ciencia Política, 4 (8), 115-146. Recuperado de: https://revistas. unal.edu.co/index.php/cienciapol/article/view/16210/17086

Rappaport, J. (2007). Intelectuales públicos indígenas en América Latina: una aproximación comparativa. Revista Iberoamericana, 73 (220), 615-630.

Rojas, R. et al. (Coords.) (2017). Breve panorama educativo de la población indígena. México: INEE. Recuperado de: http://publicaciones.inee.edu.mx/buscadorPub/P3/B/107/P3B107.pdf

Rosado-May, F. (2017). Formación universitaria intercultural para indígenas mayas de Yucatán. Anthropologica, (39), 215-242. Recuperado de: revistas.pucp. edu.pe/index.php/anthropologica/article/download/16511/19634

Salmerón, F. (2017). Prólogo. En González, F. Rosado-May, F. \& Dietz, G. (2017). La gestión de la Educación Superior Intercultural en México (pp. 5-18) México: Universidad Autónoma de Guerrero A.C.

$\mathrm{UNICH}$ (2010). Intercambio de experiencias laborales de los egresados de la primera generación de la UNICH. Recuperado de: http:/ / www.unich.edu.mx/primer-forode-egresados/691/

Zapata C. \& A. Rojas (2017). Formación de intelectuales mapuche en Chile: el caso del programa de becas de la Fundación Ford. Andamios (14) 33, 325-345 http://www.scielo.org.mx/pdf/anda/v14n33/1870-0063-anda-14-33-00325. pdf 
